\title{
EEG Signal Enhancement Using OWA Filter
}

\author{
Soham Yadav ${ }^{1}$, Jeevika Pawar $^{1}$, Girish Patil ${ }^{1}$, and Shivangi Agarwal ${ }^{1}$ \\ ${ }^{1}$ Department of Electronics Engineering, Ramrao Adik Institute of Technology, Nerul, Navi Mumbai, 400706, India
}

\begin{abstract}
Biomedical signal monitoring and recording are an integral part of medical diagnosis and treatment control mechanisms. For this, enhanced signals with appropriate peak preservation are required. The OWA (Ordered Weighted Aggregation) Filter used in this paper helps in non-linear signal filtering and preservation of peaks for accurate medical diagnosis. Weights are an important aspect of the OWA filter, the Gaussian method and the KDE (Kernel Density Estimation) function are used to obtain a precise output which helps in filtering the signal. This filter is further compared with another non-linear filter that is the median filter to understand the compatibility and the preciseness of the filter in a much deeper sense.

OWA | filter | peak | kernel density estimation | probability density | EPD (Estimated Probability Density)
\end{abstract}

\section{Introduction}

When it comes to signal filtering, noise reduction is a crucial aspect. Filtering biomedical signals should be done with extreme caution. The need for effective noise reduction must be balanced against the signal's need for as little filtration as possible. Filtering wave-forms like the electroencephalogram signal (EEG) or the electrocardiograph signals (ECG) should be given special attention. In this paper we consider the EEG signals for signal filtering and enhancement. This paper reflects on the refinement of signals. Noise filtering, smoothing, and other signal improvement methods are also used to improve the analytical signals' signal-to-noise ratio. In order to remove unwanted noise from the signal, a careful selection of the experimental conditions and a detailed review of the sources of noise should be developed as the first approach.[1] [2]

There are times where for instance, smoothing of the analytical signal is needed to evaluate or validate the presence of a peak in a noisy baseline. Any peak narrowing or deconvolution techniques often involve simultaneous filtering of the noise. Filtering can aid in the visual identification of the signal's significant characteristics. For such signal filtering and enhancement, we use a novel nonlinear filter called the OWA filter. OWA filter is better known for its precise measurements and preservation of peak signals, through this paper we venture the field of signal enhancement using the OWA filter.[3] [4] [5]

\section{Literature Survey}

OWA operators were first introduced by Ronald R. Yager in 1988[6] and have been subjected to numerous research studies later on. Since its appearance, it has been used in a wide variety of different applications that include decision making, neural networks, database systems, fuzzy logic controllers, expert systems, learning systems, market research, linguistic quantified propositions and mathematical programming [7]. It is a parameterised class of mean type aggregation operators [9]. If the weights in OWA operator are determined, then special aggregation operators can be obtained, such as arithmetic average, max and min operators, etc. An application of OWA operator to suppress noise in ECG signal processing is presented in Pander (2017)[9]. Along with this, a new approach to robust filtering with OWA aggregation operator is proposed. The process of using OWA operators in signal filtering has come to light.

\section{OWA Filter}

Filters are used to remove unwanted frequencies from the signal. But the normal subtraction of the noise or the unwanted frequencies from the original signal might lead to the loss of some important data of the signal required. This loss can compromise the accuracy of the signal, which may affect the results. OWA (Ordered weighted Aggregation) filter is used to overcome this data loss, by processing the weighted average of the ordered data from the signal in a moving window. The process of OWA filter is as follows, the 1 st step is to "rearrange the signal data" in the descending order, step 2 is finding the weights of the signal data (Eq 5) and the step 3 is to find the mean type aggregation by calculating the weights and the data points [6].

$$
Y i=\sum_{i=-N}^{N} W i X i
$$

where. $\mathrm{Xi}$ denotes the discrete input signal and $\mathrm{Yi}$ denotes the filtered output, Wi denotes the weights calculated, and $\mathrm{i}=(1 \ldots . . \mathrm{M})$. [7] [9] The window length "M" will 
be asked from the user and the window will iterate over the set of data points in the signal.

$$
O W A(Y 1 \ldots . . . Y n)=\sum_{i=-N}^{N} W i X i
$$

The " $M$ " denotes the window length where $\mathrm{M}=$ $(2 \mathrm{~N}+1)$ and $\mathrm{i}$ is the range of the elements in a window, where $\mathrm{i}=(1 \ldots \ldots \mathrm{M})$. The operation of OWA is parameterised by the choice of weights. Assuming, if the weight wi $=1 / \mathrm{M}$, where $\mathrm{i}=(1 \ldots \mathrm{M})$, [7] [9]

$$
O W A(Y 1 \ldots . . . Y n)=\frac{1}{M} \sum_{i=-N}^{N} X i
$$

It turns into a simple averaging filter. The main operation of the OWA depends on the aggregation of the signal based on the weights of its data points. If the summation of all the weights of the signal elements is;

$$
\sum_{i=-N}^{N} W i=1
$$
filter.

$\mathrm{i}=(1 \ldots \mathrm{M})$, for the appropriate working of the OWA

\subsection{Flowchart}

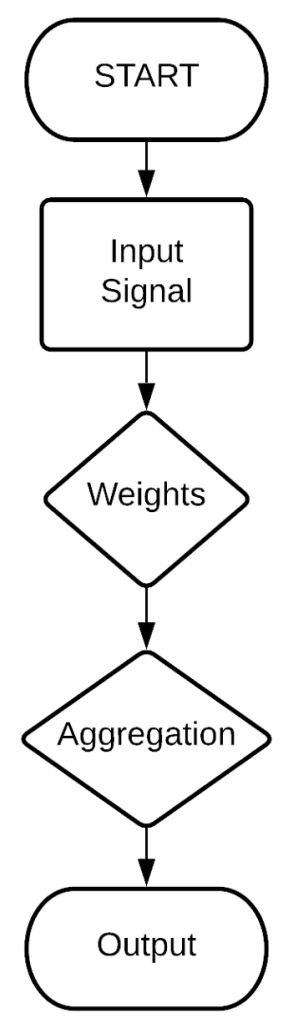

Figure 1. Flowchart of OWA Filter Process
- Any signal can be used as an input, here we have used an EEG signal as an input.

- Finding weights is the most important part, weights are found based on the importance or the value of the data points.

- Then the data points are aggregated based on their weights and output is generated.

\section{Weights}

All the data points are allotted with weights which gives the summation as one. There are many ways for finding weights of the data points, one such method is:

- Kernel Density Estimation:

Kernel Density Estimation (KDE), is introduced to estimate the probability density function (Eq 6,7). It effectively captures the complex data distribution characteristic of input values without using complicated classification methods .

$$
W j=\frac{P(V j)}{\sum_{l=1}^{n} P(V l)}
$$

\section{Kernel Density Estimation}

Determining weights of the input data is one of the most important part of the OWA filter. The process of finding weights is very confusing and one of the most searched topics. Many different processes have been suggested among which Kernel Density gives a precise value for determination of weights. In other processes, the weights are found by using common ways, neglecting the complex data and without considering their distribution characteristics, kernel density over comes these draw backs. Since there is no practical demonstration of use of KDE for biomedical signal processing, this paper demonstrates how KDE can be Used practically.

EPD function is an essential part of KDE. This mathematical tool can be used to find the probability density which helps to describe the distribution of input values.

$$
P(v)=\frac{1}{n h} \sum_{i=1}^{n} K\left(\frac{v-v i}{h}\right)
$$

where $\mathrm{p}(\mathrm{v})$ denotes the EPD function of random variable(v), $\mathrm{n}$ is the input value, $\mathrm{h}$ is the smoothing parameter also known as bandwidth, " $\mathrm{K}$ " is the kernel [9]. The accuracy of EPD depends on the smoothing factor and the kernel. Using the EPD and kernel we can find weights of the input signal.

$$
W j=\frac{P(V j)}{\sum_{l=1}^{n} P(V l)}=\frac{\frac{1}{n h} \sum_{i=1}^{n} K\left(\frac{v-v i}{h}\right)}{\sum_{i=1}^{n}\left(\frac{1}{n h} \sum_{i=1}^{n} K\left(\frac{v-v i}{h}\right)\right)}
$$

where $\mathrm{j}$ denotes the maximum element of an array of data, $\mathrm{Wj}$ is the maximum weight where $\mathrm{j}=(1 \ldots . \mathrm{M})$, similarly $\mathrm{Vj}$ is the maximum input data and $\mathrm{p}$ denotes the probability density. $1=(1 \ldots \mathrm{n})$ and $\mathrm{Vl}$ is $(\mathrm{V} 1 \ldots . . . \mathrm{Vn})$. The summation of all the weights; i.e. $\sum_{i=-N}^{N} W i=1$. 


\section{SNR and FFT}

\subsection{SNR}

SNR calculations may either be easy or complicated, and it depends on the devices in question and obtainable knowledge. If the SNR measurements are in $\mathrm{dB}$, then you can deduct the noise amount from the specified signal: SNR = $\mathrm{S}-\mathrm{N}$. The distinction within the numbers equals the SNR. as an example, considering a radio wave with a strength of -10 sound unit and a noise signal of -50 sound unit. Therefore, $-10-(-50)=40 \mathrm{~dB}$.

As shown explicitly earlier, shrewd SNR may be concerned, as well. So, for complicated calculations, divide the worth of the specified signal by the quantity of the noise, taking logarithm of the result into consideration, i.e., $\log (\mathrm{S} / \mathrm{N})$. After this, if the signal strength measurements are in watts (power), then multiply by twenty. However, if the units are in voltage, then may be multiplied by ten. Furthermore, for power, $\mathrm{SNR}=20 \log (\mathrm{S} / \mathrm{N})$ and for voltage, $\mathrm{SNR}=10 \log (\mathrm{S} / \mathrm{N})$. Also, the ensuing calculation is that the SNR is in $\mathrm{dB}$. as an example, measured noise worth $(\mathrm{N})$ is $2 \mathrm{~V}$, and signal $(\mathrm{S})$ is $300 \mathrm{mV}$. The SNR is $10 \log (0.3 / 0.000002)$ or around $62 \mathrm{~dB}$.

\subsection{FFT}

FFT of Fourier analysis converts a signal from original domain of that signal into frequency domain or it converts the frequency domain into time domain.

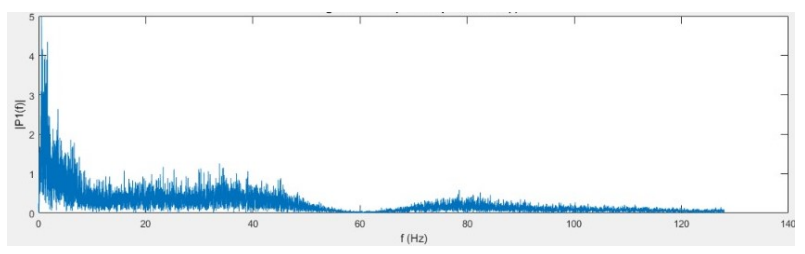

Figure 2. FFT of Input Signal

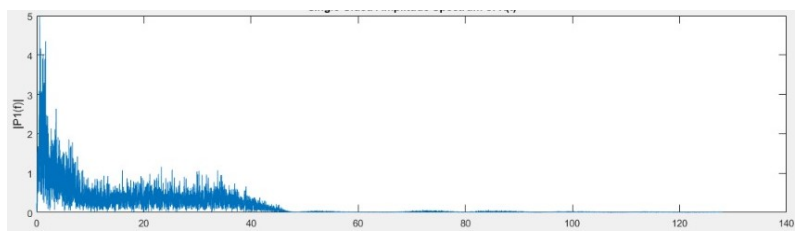

Figure 3. FFT of Output Signal

\section{Median Filter}

It is a non-linear filter and is simple in comparison to other non-linear filters and hence widely used. The process of median filter is as follows, Step 1: is to arrange the number in numerical order, in step 2: the input data is counted and in step 3 in case 1 if the count is odd, the input data is divided by 2 , where as in case 2 if the count is even then the average of the input data and the next higher data is calculated.

$$
\begin{gathered}
\text { Case } 1: \frac{X i}{2}=Y i(\text { absolutevalue }) \\
\text { Case } 2: \frac{X i+(X i+1)}{2}=Y i
\end{gathered}
$$

where $\mathrm{Xi}$ is the data points and $\mathrm{Yi}$ is the filtered output, $\mathrm{i}=(1 \ldots \mathrm{n})$

\section{Software}

MATLAB (an abbreviation of "matrix laboratory") is a multi-paradigm programming language and a numeric computing environment developed by MathWorks. MATLAB allows matrix manipulations, plotting of functions and data, implementation of algorithms, creating user interfaces, and interfacing with programs written in other languages. It is used to write special-purpose language that can be used to write moderate-size programs that can solve problems involving the manipulation of numbers. MATLAB is used in a wide variety of domains from the natural sciences, to engineering, to finance, and beyond, and it is heavily used in industry. In this paper MATLAB has been used for data implementation and simulation of the OWA filter. An OWA program was created using the general OWA formulas and implementation of the KDE to find out weights which were then used in filtering of the signal, finding the SNR and also for Peak Preservation. The use of MATLAB makes it easy to generate complex outputs, compare multiple filters, their features, etc.

\section{Comparison}

\begin{tabular}{cccc}
\hline Sr.No. & Parameters & OWA Filter & Median Filter \\
\hline 1 & SNR & $25.586 \mathrm{db}$ & $22.213 \mathrm{db}$ \\
2 & Peaks & 0.05960139583 & 0.4412551886 \\
\hline
\end{tabular}

Table 1. Comparison of OWA and Median Filters

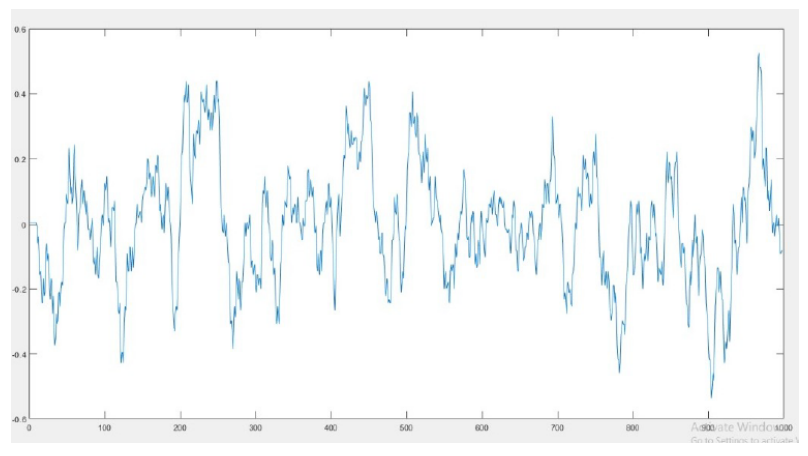

Figure 4. OWA Filtered Output 


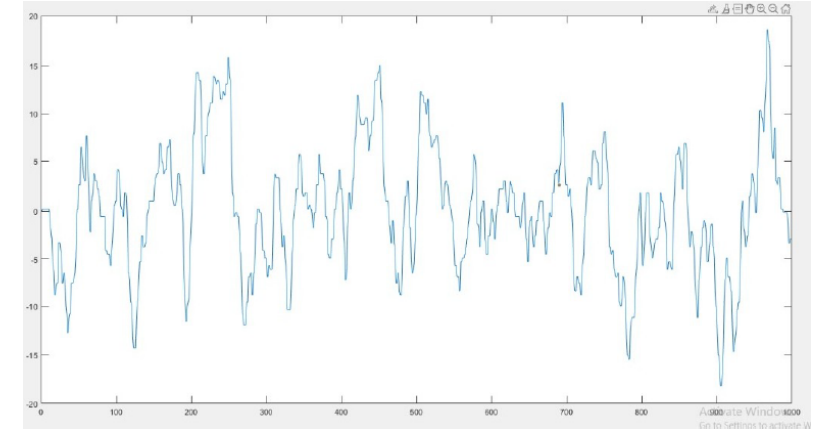

Figure 5. Median Filtered Output

\section{Results and Analysis}

From the signal to noise ratios of different EEG signals we can figure out that the OWA filter is capable of filtering signal while maintaining peaks without losing any data.

\begin{tabular}{|c|c|c|c|}
\hline Sr no. & Chb Types & OWA SNR & Median SNR \\
\hline 1 & Chb0101 & $24.628 \mathrm{db}$ & $22.765 \mathrm{db}$ \\
\hline 2 & Chb0102 & $23.071 \mathrm{db}$ & $18.453 \mathrm{db}$ \\
\hline 3 & Chb0103 & $19.641 \mathrm{db}$ & $17.098 \mathrm{db}$ \\
\hline 4 & Chb0201 & $24.367 \mathrm{db}$ & $21.876 \mathrm{db}$ \\
\hline 5 & Chb0202 & $21.351 \mathrm{db}$ & $21.021 \mathrm{db}$ \\
\hline 6 & Chb0203 & $22.439 \mathrm{db}$ & $20.854 \mathrm{db}$ \\
\hline 7 & Chb0301 & $16.432 \mathrm{db}$ & $15.583 \mathrm{db}$ \\
\hline 8 & Chb0302 & $18.986 \mathrm{db}$ & $18.637 \mathrm{db}$ \\
\hline 9 & Chb0303 & $19.942 \mathrm{db}$ & $15.734 \mathrm{db}$ \\
\hline 10 & Chb0401 & $21.895 \mathrm{db}$ & $17.872 \mathrm{db}$ \\
\hline 11 & Chb0402 & $18.678 \mathrm{db}$ & $14.093 \mathrm{db}$ \\
\hline 12 & Cbh0403 & $26.554 \mathrm{db}$ & $24.476 \mathrm{db}$ \\
\hline 13 & Chb0501 & $19.734 \mathrm{db}$ & $15.089 \mathrm{db}$ \\
\hline 14 & Chb0502 & $17.318 \mathrm{db}$ & $16.347 \mathrm{db}$ \\
\hline 15 & Chb0503 & $17.648 \mathrm{db}$ & $14.987 \mathrm{db}$ \\
\hline
\end{tabular}

Table 2. Signal to Noise Ratio

\begin{tabular}{|c|c|c|c|}
\hline Sr no. & Chb Types & OWA Peaks & Median Peaks \\
\hline 1 & Chb0101 & 0.0615 & 0.0453 \\
\hline 2 & Chb0102 & 0.0642 & 0.0519 \\
\hline 3 & Chb0103 & 0.0665 & 0.0521 \\
\hline 4 & Chb0201 & 0.0672 & 0.0485 \\
\hline 5 & Chb0202 & 0.0691 & 0.0213 \\
\hline 6 & Chb0203 & 0.0750 & 0.0691 \\
\hline 7 & chb0301 & 0.0623 & 0.0563 \\
\hline 8 & Chb0302 & 0.0894 & 0.0474 \\
\hline 9 & Chb0303 & 0.0973 & 0.0703 \\
\hline 10 & Chb0401 & 0.0566 & 0.0274 \\
\hline 11 & Chb0402 & 0.0611 & 0.0469 \\
\hline 12 & Cbh0403 & 0.0644 & 0.0295 \\
\hline 13 & Chb0501 & 0.0776 & 0.0603 \\
\hline 14 & Chb0502 & 0.0631 & 0.0576 \\
\hline 15 & Chb0503 & 0.0576 & 0.0289 \\
\hline
\end{tabular}

Table 3. Peaks Preservation of OWA Filters

The design and the algorithm used for the designing of the OWA filter specifies that the peak preservation of this filter is the most eminent and distinguished feature. The peak preservation is expected to preserve the important data which denotes the presence of SSPE (Subacute Sclerosing Panencephalitis). The presence of SSPE is only and only detected because of its peaks and specific time intervals and OWA helps in capturing the peaks efficiently. In preserving the peaks, even a minor variance in the output can turn fatal at times. For such instances, OWA can be put to use as it has the highest peak preservation. The output of this filter was studied by extracting the peak data points from the input as well from the output and were cross verified using a MATLAB function "Goodness of fit". The output of this function is in the table given below. 


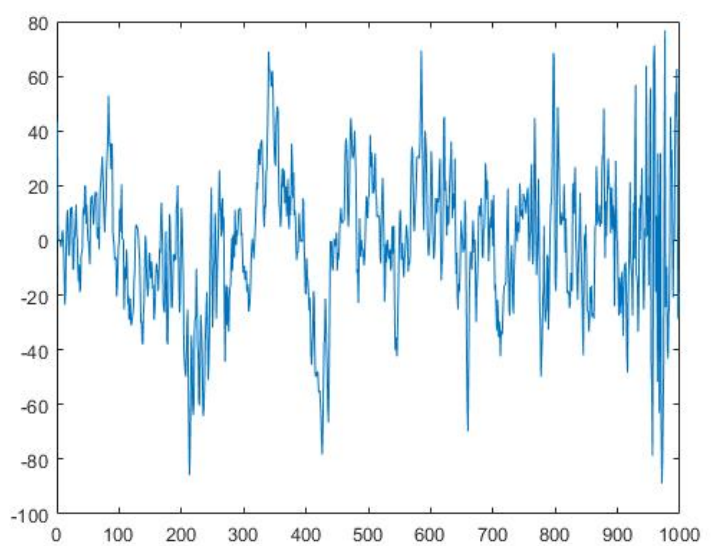

Figure 6. chb01-01 OWA Output

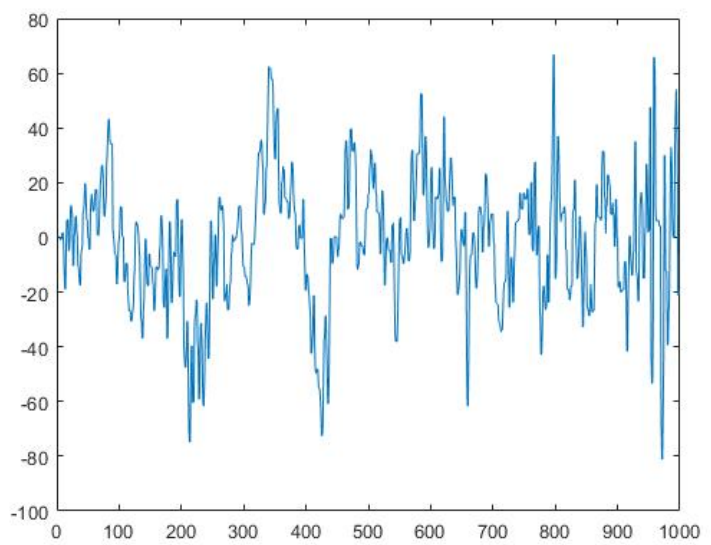

Figure 7. chb01-01 Median Filter Output

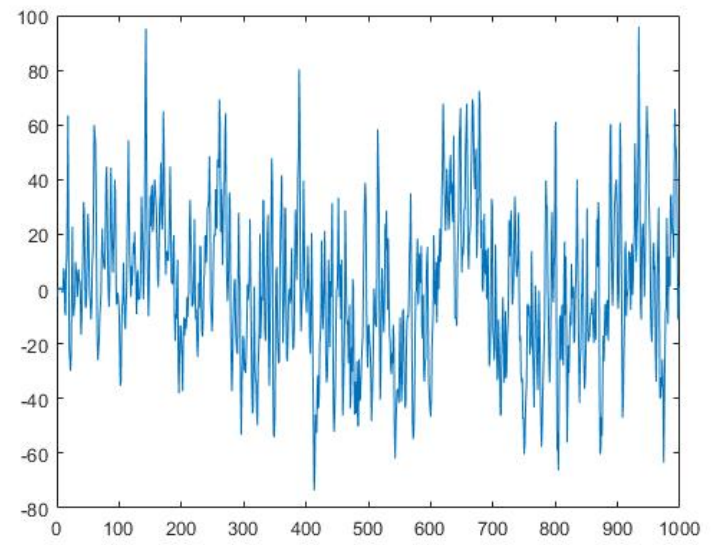

Figure 8. chb02-02 OWA Output

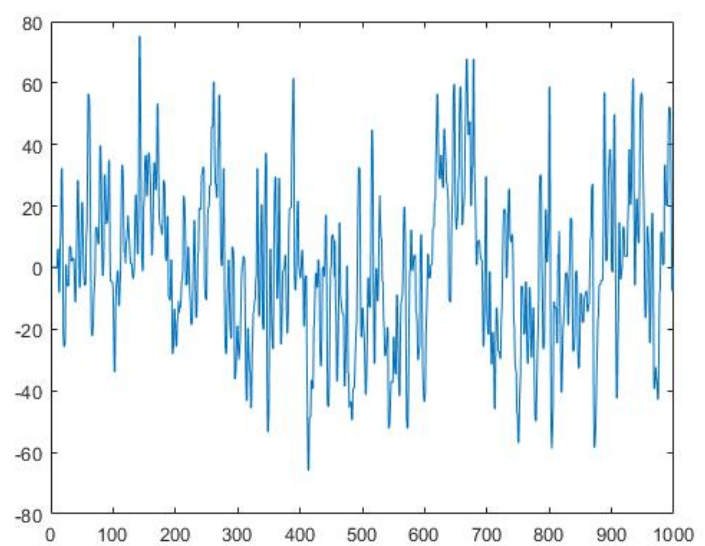

Figure 9. chb02-02 Median Filter Output

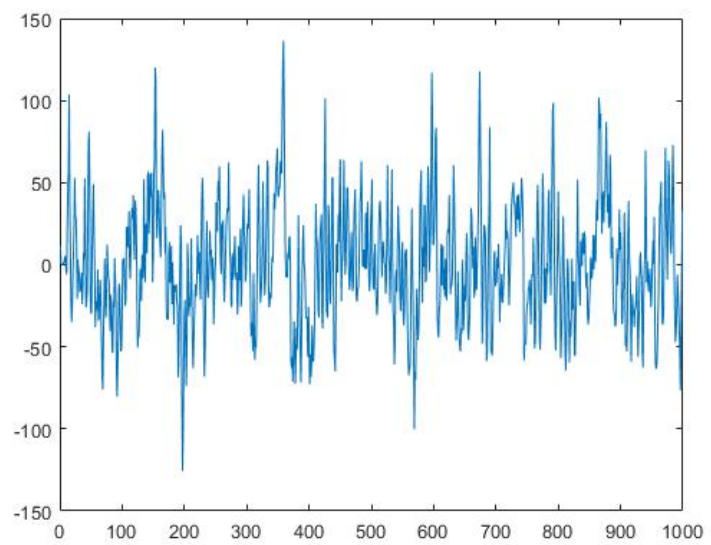

Figure 10. chb02-03 OWA Output

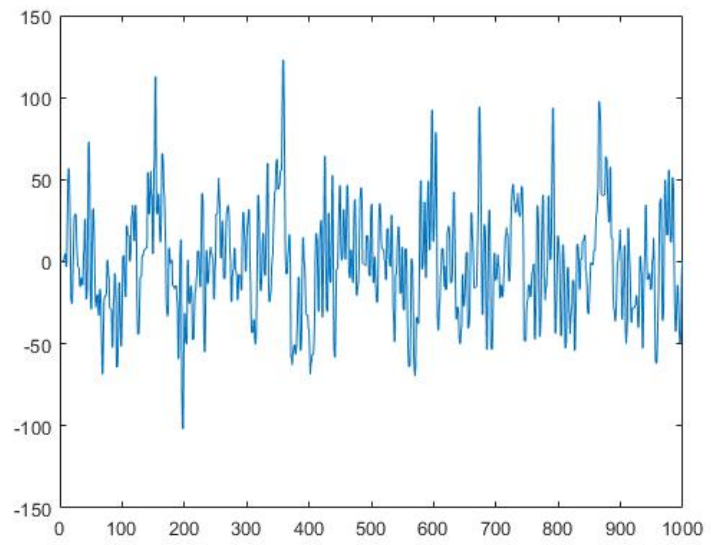

Figure 11. chb02-03 Median Filter Output 


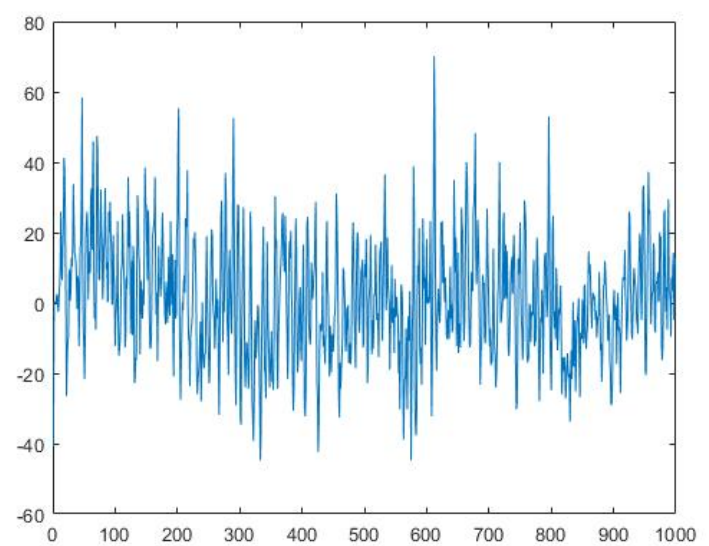

Figure 12. chb03-03 OWA Output

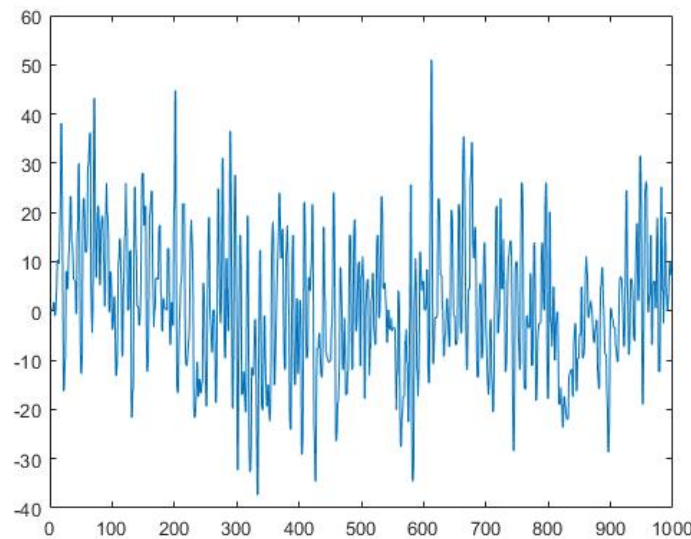

Figure 13. chb03-03 Median Filter Output

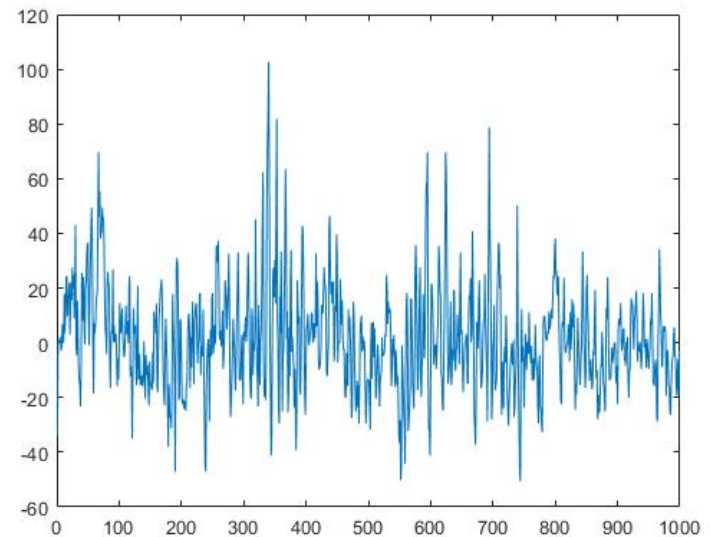

Figure 14. chb04-01 OWA Output

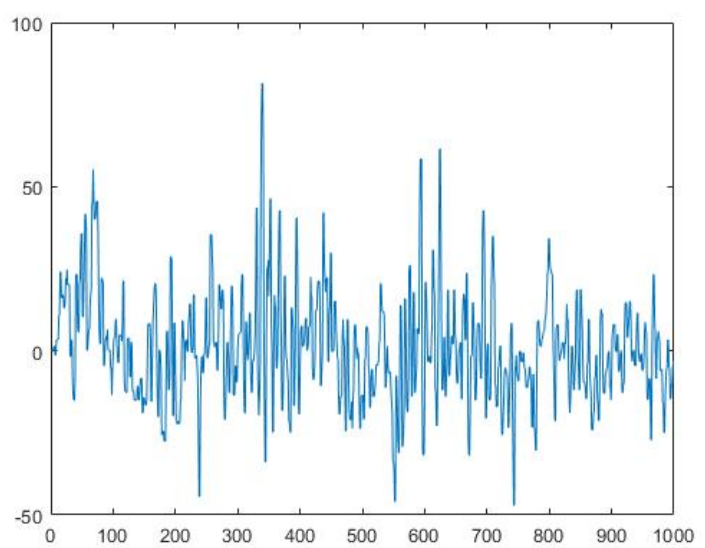

Figure 15. chb04-01 Median Filter Output

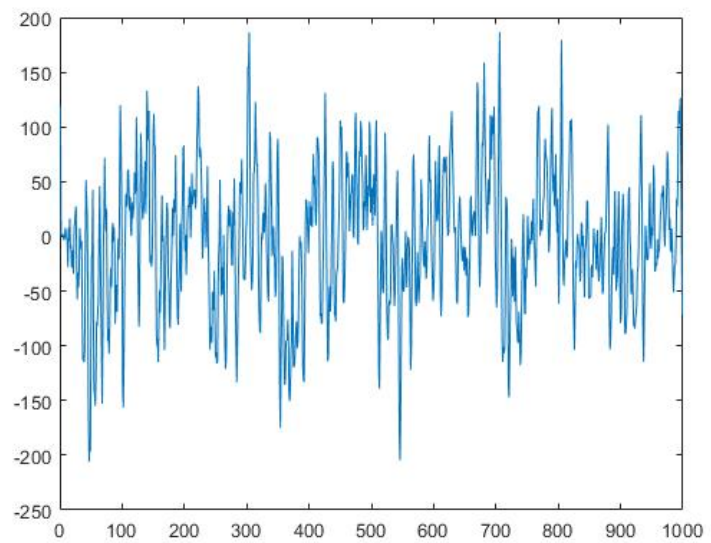

Figure 16. chb05-02 OWA Output

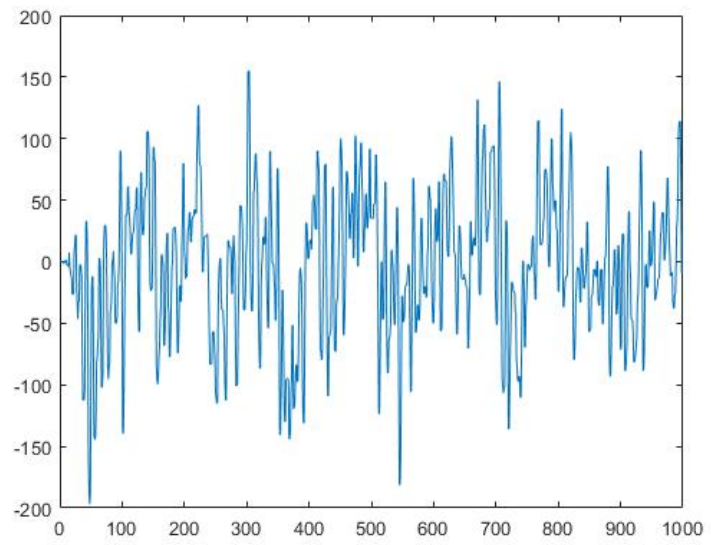

Figure 17. chb05-02 Median Filter Output 


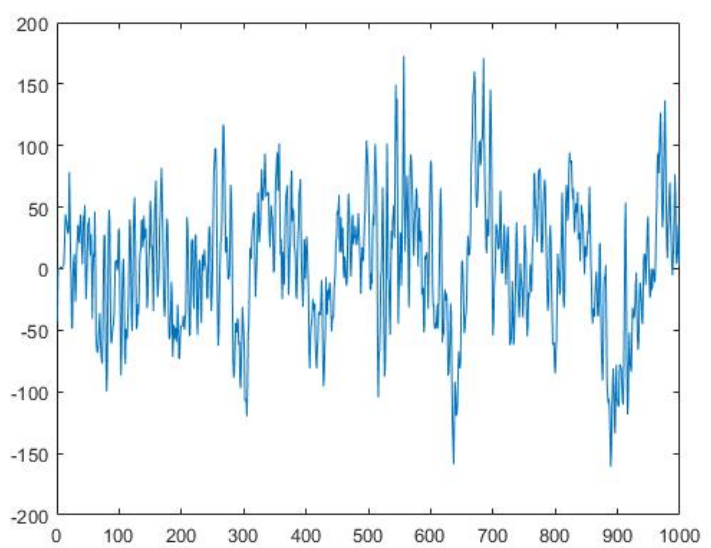

Figure 18. chb05-03 OWA Output

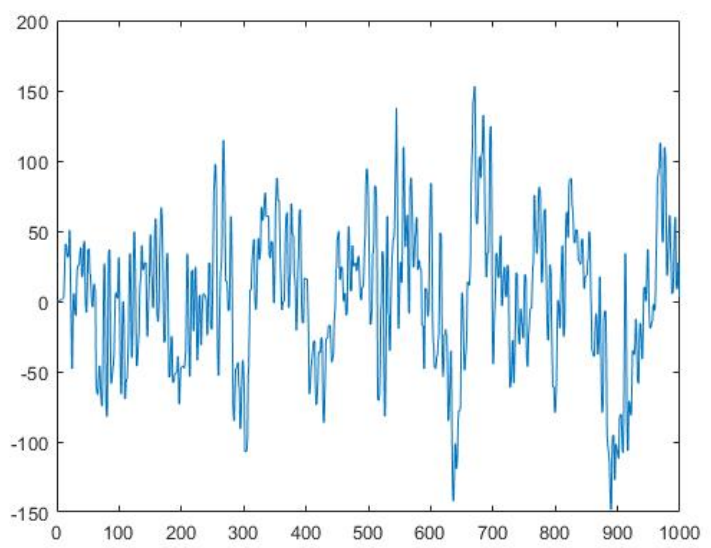

Figure 19. chb05-03 Median Filter Output

\subsection{Result and discussion}

This paper lights a torch on differentiating between the SSPE signals and normal epilepsy signals. The OWA filter is considered more accurate for biomedical signal processes. The signal is applied as an input to the filter and the weights are assigned to the data based on their value. The data is then aggregated on the basis of their weights and an output is obtained. While considering the window length for the signal, the window should be small and odd in number to increase the accuracy of the output filter. The appropriate frequency for this given filter is $60 \mathrm{~Hz}$ and the sampling frequency is 260 . The SNR is obtained by con- structing a formula which provides the ratio of signal to noise.

\section{Conclusion}

EEG signals should be filtered in real time without losing their original form. To ensure this, OWA filter was used and implemented and compared with a median filter to obtain the output signal as close to its original form as possible. It is shown here OWA is better non-linear filter compared to other non-linear filters by comparing their SNR values, peak preservation and other aspect of the signal.

\section{References}

[1] Schaefer, P.A. and Mitchell, H.B., 1999. A generalized OWA operator. International Journal of Intelligent Systems, 14(2), pp.123-143.

[2] Mianabadi, H., Sheikhmohammady, M., Mostert, E. and Van de Giesen, N., 2014. Application of the ordered weighted averaging (OWA) method to the Caspian Sea conflict. Stochastic environmental research and risk assessment, 28(6), pp.1359-1372

[3] Torra, V., 1997. The weighted OWA operator. International Journal of Intelligent Systems, 12(2), pp.153166.

[4] Xu, Z., 2005. An overview of methods for determining OWA weights. International journal of intelligent systems, 20(8), pp.843-865.

[5] Lin, M., Xu, W., Lin, Z. and Chen, R., 2020. Determine OWA operator weights using kernel density estimation. Economic Research-Ekonomska Istraživanja, 33(1), pp.1441-1464.

[6] Yager, R.R., 1988. On ordered weighted averaging aggregation operators in multicriteria decisionmaking. IEEE Transactions on systems, Man, and Cybernetics, 18(1), pp.183-190.

[7] Pander, T., 2019. EEG signal improvement with cascaded filter based on OWA operator. ignal, Image and Video Processing, 13(6), pp.1165-1171.

[8] Pander, T. and Przybyla, T., 2018, June. ECG signal enhancement with serial cascade OWA filter. In2018 25th International Conference" Mixed Design of Integrated Circuits and System"(MIXDES), (pp. 366-370). IEEE.

[9] Pander, T. and Wróbel, J., 2017, June. OWA aggregation operator in robust filtering. In IEEE 2017 MIXDES-24th International Conference" Mixed Design of Integrated Circuits and Systems, (pp. 532-536). IEEE. 